\title{
Patterns of non-rhegmatogenous elevations of the retina
}

\author{
HARVEY LINCOFF \\ New York \\ A N D \\ INGRID KREISSIG \\ Bonn, Germany
}

The purpose of this paper is to point out the differential diagnostic value of contour and topography in distinguishing a number of non-rhegmatogenous elevations of the retina. The other diagnostic aspects of the disorders that are well known will be neglected for the sake of brevity.

The characteristic contours of rhegmatogenous detachment were described in an earlier communication (Lincoff and Gieser, 1971). In general, rhegmatogenous detachments are progressive, become extensive, and spread from ora to disc. They develop consistent dependent shapes in relation to the position of the most superior retinal break and have regular convex borders and surfaces. Detachments from superior holes develop bullae; detachments from inferior holes do not. Pigment lines develop at stationary borders. In contrast, non-rhegmatogenous elevations of the retina tend to be confined, to be either peripheral or central, and not to extend from ora to disc. Borders are often irregular and sometimes concave. Pigment lines are exceptional. Dependent bullous shapes develop in exudative disorders but they are peculiar enough to be distinguished from rhegmatogenous detachment. The contours of non-rhegmatogenous retinal elevations are mostly distinct enough to conclude that a retinal break is not present.

Non-rhegmatogenous elevations may be classified topographically as follows:
(a) Peripherul
(1) Cystoid degeneration (Straatsma and Foos, 1973)
(2) Degenerative retinal schisis
(3) Oedema
(b) Central
(I) Central serous retınopathy
(2) Disciform degeneration
(3) Macular cyst
(4) Ischaemic oedema
(5) Traction detachment
(6) Tractional schisis 
(c) Dependent (exudative detachments)

(i) Tumour

(ii) Uveitis

(iii) Pigment epitheliopathy

(a) Peripheral retinal elevations

(I) GYSTOID DEGENERATION

This commonly occurring retinal elevation in the periphery is not often a problem for differential diagnosis. It is a shallow elevation, confined to the peripheral retina, with a characteristic stippled or cystoid appearance. It progresses slowly or not at all. Rarely does it extend to the equator, and it never advances to the disc. Its border tends to be scalloped, irregular, and occasionally concave (Fig. I).

It becomes of diagnostic concern when holes are found in the internal layer of a particular scallop. The question arises whether the holes are full-thickness and the elevation around them an early rhegmatogenous detachment requiring treatment. Biomicroscopy usually cannot ascertain the presence or absence of an external retinal layer. The absence of a dependent contour in relation to the holes and the failure of the elevation to progress indicate that the holes are not functioning and probably exist only in the internal layers. The absence of a pigment demarcation line when the elevation is first seen distinguishes it from an arrested peripheral detachment; the failure to develop a pigment demarcation line over several months of observation tends to confirm that only the inner layers of the retina are elevated.

\section{(2) SGHISIS}

Retinal schisis is more of a problem for differential diagnosis because it more closely resembles rhegmatogenous detachment. The cyst formations in advanced schisis have unbroken convex contours and may be somewhat bullous. Their transparency suggests a cystic origin, but atrophic retina in a rhegmatogenous detachment of long standing has a similar transparency. While cysts may be extensive, even beyond the equator when first seen, they move slowly or not at all. Occasionally a white glial line develops at the peripheral border of an old cyst, but pigmented demarcation lines do not occur unless the external layer detaches.

The shape of most cysts is diagnostic. Retinal cysts tend to be circular; they have a characteristic tenseness like a taut balloon; they lack folds and neither undulate with motion nor shift with a change in position. Occasionally the peripheral border of a cyst may be only at a tangent to the ora serrata, and a full circle is revealed. But even when the

F I G. I Peripheral cystoid degeneration with transitional schisis. Irregular posterior border is scalloped and occasionally concave. There are two holes in the internal layers at $\mathrm{I} .30$ o'clock.

FI G. 2 Peripheral degenerative schisis. Two circular retinal cysts with holes in the internal layers. Broken line indicates change in contour that might be expected if a break in the external layer should occur and detachment ensue. FI G. 3 Peripheral oedema. Circumferential oedema in the periphery after blunt trauma to the eye, with choroidal elevation and posterior extension of retinal oedema infero-nasally. A localized pocket of fluid exists along the inferotemporal vessels.

FI G. 4 Macular cyst with a hole. The symmetry about the hole suggests that the break is in the internal layers of the retina only.

F I G. 5 Ischaemic oedema of retina after closure of superior branch of retinal artery. The posterior border is straight to the macula and along the temporal raphe. The anterior border stops short of the ora serrata. 

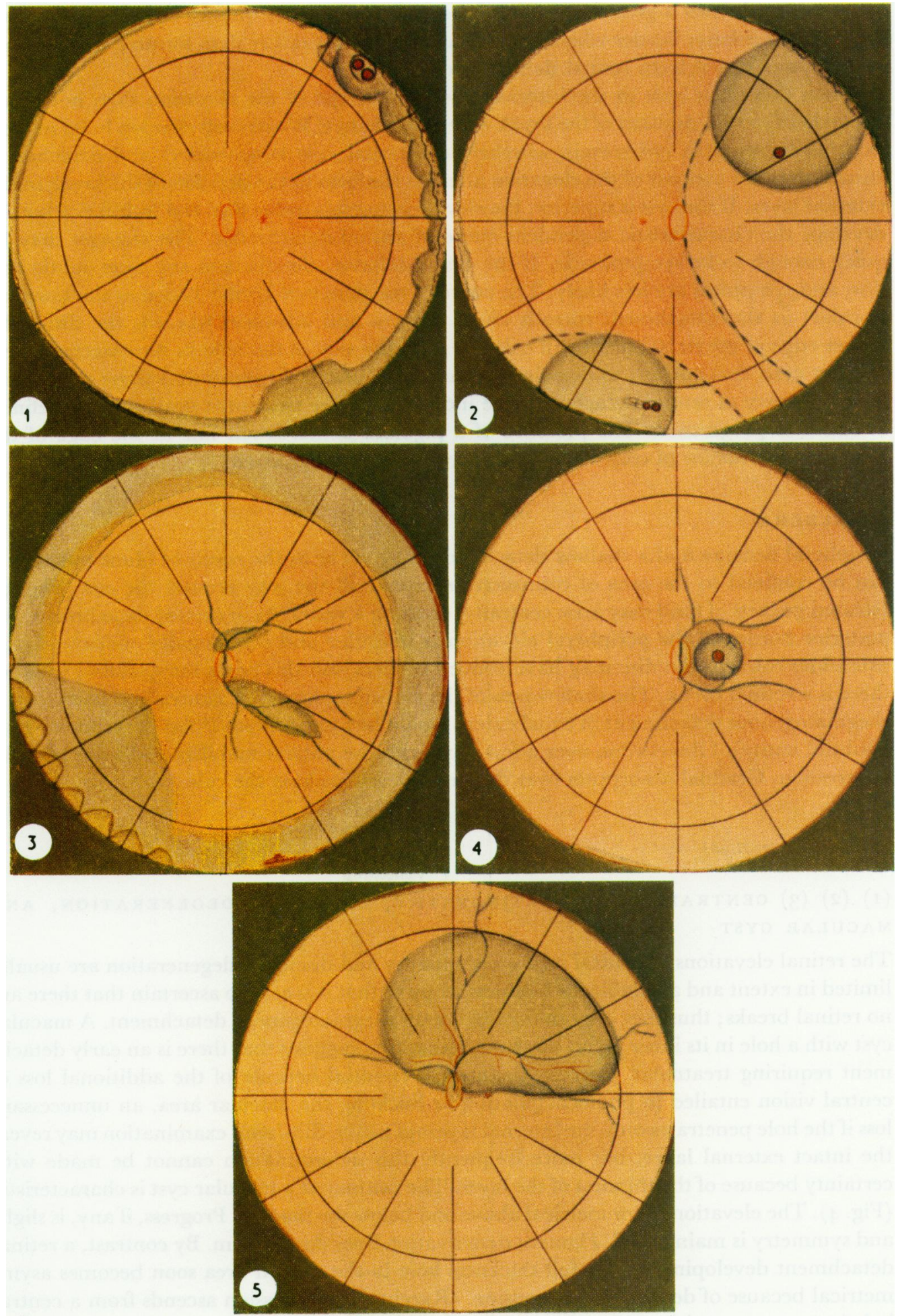

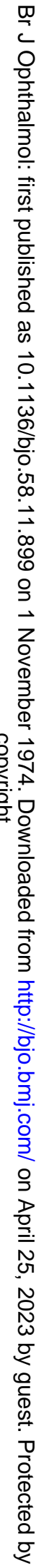


ora serrata subtends a greater portion of the cyst, the circular quality of the borders, particularly the inferior border which curves back upon itself rather than forming a dependent arc, distinguishes it from retinal detachment (Fig. 2).

When there is a hole in the internal layer, the shape of the elevation does not relate dependently to the position of the break except by chance. When cysts develop holes in their external layers (it is not certain whether an internal hole is necessary) and detachment ensues, then the contour of the elevation alters to conform to the position of the break in the external layer. If the cyst is superior, the elevation spreads to the disc and its inferior border develops the characteristic dependent curve of retinal detachment. The changes may be quick enough to be symptomatic. If the cyst is inferior, the changes are more subtle and slow and symptoms are less likely. The curve of the posterior border becomes shallow (less circular) as the detachment spreads along the ora and advances towards the disc. The higher edge of the detachment will coincide with the side of the hole in the external layer. Inferior cysts that convert to detachments are problems in diagnosis and those embarking on the surgery of retinal detachment are inclined to defer treatment until there is definite evidence of progression. The presence of a pigment demarcation line at the edge of the elevation is evidence of conversion; successive lines of progression.

\section{(3) OEDEMA}

Peripheral oedema and a shallow detachment may occur as the result of blunt trauma. It can be confined to the area of contusion or extend all the way around the eye (Fig. 3). Isolated pockets of fluid may arise centrally, or along a vessel. As in cystoid detachment, the posterior border of the peripheral elevation is irregular and occasionally concave. Local extensions towards the disc may have convex borders and this poses the question whether there is a retinal break. The shallowness of the elevation, however, contradicts its extent; a rhegmatogenous detachment of equal extent would be more bullous ("flat detachment" is a doubtful entity). Observation over a few days with the patient mobilized will not provoke progression. Gradual absorption over days or several weeks is the rule.

\section{(b) Central elevations}

(I)（2）（3） Gentral Serous Retinopathy， Disgiform Degeneration，AND MACULAR GYST

The retinal elevations in central serous retinopathy and disciform degeneration are usually limited in extent and available for biomicroscopy so that it is easy to ascertain that there are no retinal breaks; thus they are not confused with rhegmatogenous detachment. A macular cyst with a hole in its inner layer, however, sometimes suggests that there is an early detachment requiring treatment. Correct diagnosis is essential because of the additional loss of central vision entailed in photocoagulating or buckling the macular area, an unnecessary loss if the hole penetrates only the internal layers of a cyst. Slit-lamp examination may reveal the intact external layer, but more frequently this determination cannot be made with certainty because of the thinness of the layer. The contour of a macular cyst is characteristic (Fig. 4). The elevation is symmetrical above and below the macula. Progress, if any, is slight and symmetry is maintained. Pigment demarcation lines do not form. By contrast, a retinal detachment developing from a full-thickness hole in the central area soon becomes asymmetrical because of dependent progression. (Rarely the progression ascends from a central hole.) 
An additional factor which warrants caution in making the diagnosis of retinal detachment secondary to a macular hole is its extremely low incidence as compared to that of macular cyst. In a decade, the retinal service at The New York Hospital has not recorded a single rhegmatogenous detachment resulting from a primary hole in the macula. Macular holes have occurred secondarily in extensive detachments, the origin of which were primary breaks in the periphery. Detachments have occurred as a result of breaks near the macula; paracentral holes are usually full-thickness. In summary, a diagnosis of rhegmatogenous detachment secondary to macular hole can probably be made with certainty only if the elevation demonstrates an asymmetry about the macula and progression.

\section{(4) ISGHAEMIG OEDEMA}

A sequence of events may follow the closure of a branch of the central retinal artery which mimics retinal detachment. For example, with closure of a superior temporal vessel, the patient will present with sudden loss of the inferior visual field, much the same as might occur after a rapidly developing superior bullous detachment. Examination reveals profound oedema of the retina along the distribution of the occluded vessel. If the artery has reopened, however, the cause of the oedema may not be apparent and the impression of a "flat detachment" of the retina may be obtained. With the direct ophthalmoscope the degree of elevation of the retina is exaggerated because the oedema obscures the choroidal markings. Binocular ophthalmoscopy reveals that the shape and topography of the retinal elevation is unique (Fig. 5). The height is inconsistent with the extent; it is too shallow for rhegmatogenous detachment. The posterior (central) border relates to the temporal raphe, and tends to be straight rather than convex. Examination of the periphery reveals that the elevation diminishes as it approaches the equator. The peripheral retina is flat.

\section{(5) TRAGTION DETAGHMENT}

Detachments caused by vitreous traction are unique in that they have concave borders and concave surfaces. They may be peripheral, reflecting traction at the vitreous base, or central; diabetic detachments tend to be central.

Diabetic traction detachments are caused by the adherence of the retina to a contracting posterior hyaloid membrane. The retinal elevation is oriented symmetrically around the points or lines of adhesion. It spreads slowly into the periphery through successive pigment demarcation lines as the posterior hyaloid membrane contracts. It does not reach the ora serrata, however, not even inferiorly. Dependent spread does not occur, probably because the quantity of subretinal fluid beneath a concave detachment has too little mass to effect a dependent dissection (Fig. 6).

In patients with central traction detachments the peripheral retina functions until the vascular tree becomes obliterated. A sudden loss of peripheral vision is usually due to the development of a retinal hole. The detachment, now rhegmatogenous, spreads rapidly to the ora serrata and becomes convex and dependent. The contour of the detachment depends on the position of the hole and the lines of traction. When the break is central, as is often the case, subretinal fluid quickly fills the bottom of the eye to the level of the break. It will rise $\mathrm{I}$ to 2 clock hours above the break, symmetrically on either side of the disc. The superior periphery may remain in place unless there is a superior line of traction to carry fluid to the top of the eye (Fig. 7).

A correct evaluation of the diabetic detachment, whether solely tractional or rhegmatogenous as well, is essential for a therapeutic approach. The concave detachment may respond to an encircling constricting operation aimed at relaxing the pull of the hyaloid on 
the posterior retina, as recommended by Okun, Johnston, and Boniuk (I97I). No effort need be spent in searching a concave retinal surface for a hole. The convex detachment, on the other hand, requires that a hole be found and closed. There is usually only one hole, probably because at the moment of the break the liquid vitreous pours rapidly through it, elevating the retina and relieving further traction upon it. Effective buckling of the break will restore the retina to its former traction configuration and the former peripheral field may be recovered.

\section{(6) TRAGTIONAL SGHISIS}

Occasionally the pull on the retina of an adherent vitreous membrane will cause the inner layers of the retina to split and become elevated in a pattern similar to a traction detachment. There are diagnostic and prognostic differences. The schisis spreads slowly and symmetrically beneath the points of traction, but pigment demarcation lines do not occur, evidence that only the inner layers of the retina are separating. Retinal tears may occur along the vessels. The failure of the elevation to spread abruptly in the presence of tears, and the persistence of a concave contour offers additional reassurance. The prognosis in cases of tractional schisis in diabetics, barring haemorrhage, is relatively good. The spread is limited and retinal detachment is less likely to occur. Over a number of years, the vasculature becomes obliterated and the elevated layers of retina disintegrate. The area may then appear as a large partial-thickness hole, orange and stippled, in flat retina (Fig. 8).

\section{(c) Dependent (exudative detachment)}

Exudative detachments of the retina occur as a consequence of intraocular tumours, pigment epitheliopathies, or inflammatory disorders such as Harada's disease, herpes zoster, and uveitis. Regardless of the aetiology, exudative detachments have characteristics of contour in common that distinguish them from rhegmatogenous detachment.

Exudative fluid characteristically gravitates to the dependent portion of the eye to collect symmetrically around the 6 o'clock meridian. It is best observed with the patient in a sitting position. If the patient is examined lying down, a small amount of fluid may escape notice because it spreads posteriorly as a shallow detachment (Fig. 9). When the amount of fluid increases and the detachment becomes bullous, a typical cleft between the bullae develops at the 6 o'clock position. The cleft enables the retina to accommodate to maximum elevation (Lincoff, 1972). An exudative detachment can become so bullous as to touch the lens. When this occurs it signifies that the detachment has dissected beneath the epithelium over the pars plana.

FIG. 6 Traction detachment of I year's duration in a diabetic. The retina is adherent to a central proliferative plaque. The surface of the retinal elevation is concave. The peripheral contour relates to traction lines, is irregular, and occasionally concave. There are numerous pigment demarcation lines.

FIG. 7 Rhegmatogenous spread of traction detachment in Fig. 6 as the result of a break in the posterior retina at $50^{\prime}$ 'clock. The detachment has become bullous (convex) and has spread to the ora.

FIG. 8 Tractional schisis in a diabetic with a break along a vessel. In the course of 10 years it neither progressed nor formed pigment demarcation lines. Eventually the internal layers disintegrated.

FIG. 9 Early inferior exudative detachment, secondary to a choroidal tumour at 9.30 o'clock. Broken lines indicate symmetrical pattern of shift with change in position.

FIG. I o Advanced exudative detachment to a choroidal tumour at 2.30 o'clock. There is a characteristic cleft at the 6 o'clock radian. The broken lines define the symmetrical shift of fluid into the upper quadrants when the head is turned from side to side. 

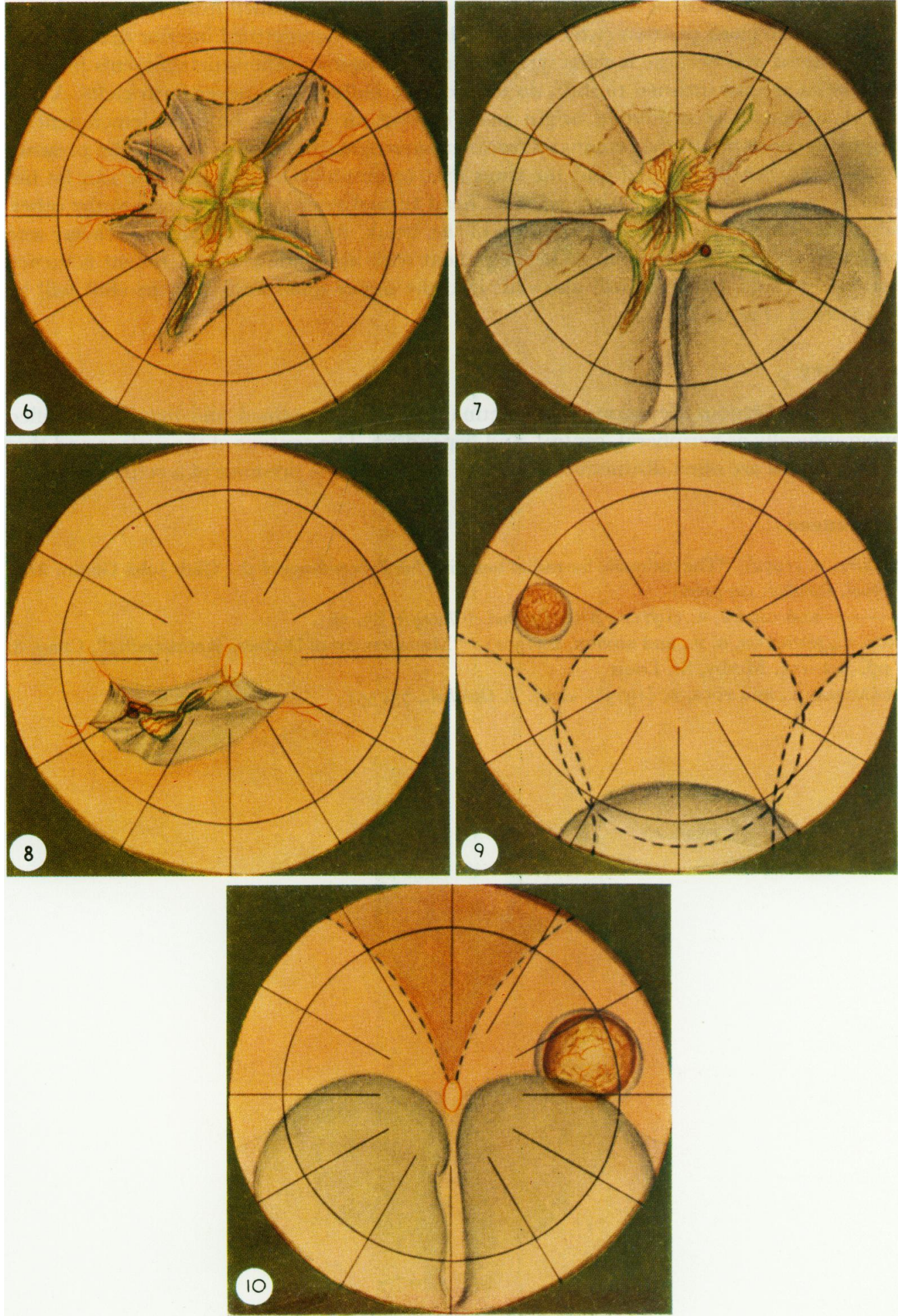
An inferior detachment of exudative origin may be distinguished from one caused by a hole at the 6 o'clock position (which would also cause a symmetrical inferior detachment) if it is bullous and because it shifts symmetrically. Rhegmatogenous detachments from inferior holes are not inclined to shift, do not become bullous, and never form clefts. A bullous detachment of exudative origin may imitate an inferior bullous detachment of rhegmatogenous origin that emanates from an unrecognized superior break. The presence of the break can be inferred and the sinus that communicates with the hole can be uncovered by examining the patient with his head turned to one side and then to the other. Subretinal fluid shifts higher on the side of the break, distending the sinus pathway and communicating with the break. When a patient with an exudative detachment is turned from side to side, the fluid will also shift into the upper quadrants, but the shift will be symmetrical (Fig. 10).

\section{Summary}

Non-rhegmatogenous elevations of the retina tend to be confined and may be classified topographically as peripheral, central, and dependent. The contour of the surface and that of the margins are often distinctive enough to contradict the presence of a retinal break.

\section{References}

LINCOFF, H. (1972) “The rationale for radial buckling" in Proceedings of the Club Jules Gonin. Mod. Probl. Ophthal. (in press)

- and GIESER, R. (1971) Arch. Ophthal. (Chicago), 85, 565.

OKUn, E., JOHNSTON, G. P., and Boniuk, I. (197I) "Managernent of Diabetic Retinopathy", Chap. 8. pp. 142-154. Mosby, St. Louis

StraAtsma, B., and Foos, R. (1973) Amer. 7. Ophthal., 75, 55 I. 